\title{
Cholesterol embolization syndrome after primary percutaneous coronary intervention in patient with acute anterior myocardial infarction is
}

\author{
Alptug Tokatli ${ }^{\mathrm{a}, *}$, Ersan Tatli ${ }^{\mathrm{b}}$, Murat Aksoy ${ }^{\mathrm{b}}$, Mehmet Akif Cakar ${ }^{\mathrm{b}}$ \\ a Golcuk Military Hospital, Department of Cardiology, Kocaeli, Turkey \\ b Sakarya University School of Medicine, Department of Cardiology, Sakarya, Turkey
}

\section{A R T I C L E I N F O}

\section{Article history:}

Received 1 May 2016

Received in revised form 21 May 2016

Accepted 22 May 2016

Available online 27 May 2016

\begin{abstract}
A B S T R A C T
Background: Cholesterol embolization syndrome is a rare and under-diagnosed clinical entity. Vascular procedures such as angiography of vascular surgery have been identified as triggering factor.

Case report: A 71-year-old man presented with acute ST segment elevated anterior myocardial infarction and coronary stenting was performed. At the clinic follow-up, coloured skin lesions were observed at both hands and feet. During follow-up, progressive deterioration in renal functions also developed gradually. Supportive therapy was provided in addition to standard treatment for myocardial infarction.

Conclusion: In conclusion cholesterol embolization syndrome should be considered in any patient presenting with cutaneous features and renal failure after invasive vascular procedure.

(c) 2016 The Society of Cardiovascular Academy. Production and hosting by Elsevier B.V. All rights reserved. This is an open access article under the CC BY-NC-ND license (http://creativecommons.org/licenses/by-nc-nd/4.0/).
\end{abstract}

\section{Introduction}

Cholesterol embolization syndrome (CES) is caused by distal embolisation of cholesterol crystals from aortic atheromatous plaques, which then result in end-organ damage. ${ }^{1}$ Herein, a case of probable CES with cutaneous manifestation on all extremities and renal failure after performing primary percutaneous coronary intervention in patient with acute anterior myocardial infarction was presented.

\section{Case report}

A 71-year-old man presented with chest pain radiating to both arms accompanied by nausea and sweating came to emergency department. He had a history of hypertension and diabetes mellitus. He was a nonsmoker and had no other remarkable history of medical condition. At the admission, his blood pressure was $100 / 60 \mathrm{mmHg}$ and heart rate was 88 beats/min. Electrocardiogram was in sinus rhythm and revealed ST segment elevation over leads V1-V6 in accordance with acute ST segment elevated anterior myocardial infarction. Echocardiography showed hypokinesia of the anterior wall, anterior septum and left ventricular apex with left ventricular ejection fraction of $48 \%$. Left ventricle end-diastolic diameter and left atrial diameter were $52 \mathrm{~mm}$ and $36 \mathrm{~mm}$,

\footnotetext{
it Peer review under responsibility of The Society of Cardiovascular Academy.

* Corresponding author at: Golcuk Asker Hastanesi, Kardiyoloji servisi, Golcuk 41910,

Kocaeli, Turkey. Tel.: +9026242602 71; fax: +9002624141111.

E-mail address: alptugtokatli@gmail.com (A. Tokatli).

Peer review under responsibility of The Society of Cardiovascular Academy.
}

respectively. However, left ventricular thrombi were not detected. In laboratory findings glucose level was $188 \mathrm{mg} / \mathrm{dl}$, HbA1c was 7.2 and total cholesterol level was $184 \mathrm{mg} / \mathrm{dl}$. Aspirin and clopidogrel were given and heparin was used for anticoagulation. He transferred to coronary angiography laboratory. Emergent coronary angiography revealed totally occluded left anterior descending (LAD) coronary artery. Circumflex and right coronary artery angiography showed non-significant stenosis. LAD lesion was crossed with guidewire and drug-eluting stent was implanted. Thrombolysis in myocardial infarction III (TIMI III) flow was observed. After the procedure the patient was transferred to coronary care unit and two days later transferred to cardiology clinic. At the clinic follow-up, painful, blue-coloured, macule lesions were observed at both hands and feet (Figs. 1, 2). These lesions became larger over the ensuing days. His blood pressure was $115 / 70 \mathrm{mmHg}$ with a pulse of 75 beats per minute, his respiration was 16 per minute and the body temperature was $36.8{ }^{\circ} \mathrm{C}$. His oxygen saturation was $96 \%$ on air and all peripheral pulses were present. During follow-up, progressive deterioration in renal functions developed gradually and his initial serum creatinine level aroused from $1.12 \mathrm{mg} / \mathrm{dL}$ to $3.02 \mathrm{mg} / \mathrm{dL}$ over the next days. Laboratory investigation showed hypereosinophilia (10\%), increased erythrocyte sedimentation rate $(68 \mathrm{~mm} / \mathrm{h})$ and normal platelet count. Serum levels of antinuclear antibody and antineutrophil cytoplasmic antibody tests were in normal range. Close chronological association with coronary intervention, the signs and the laboratory results suggested the diagnosis of probable CES. Anti-inflammatory therapy or corticosteroid therapy was not started due to the acute myocardial infarction. Antiplatelet therapy was maintained. High dose statin therapy with rosuvastatin $40 \mathrm{mg}$ /day was continued. Supportive therapy was provided by effective hydration and wound care. At 

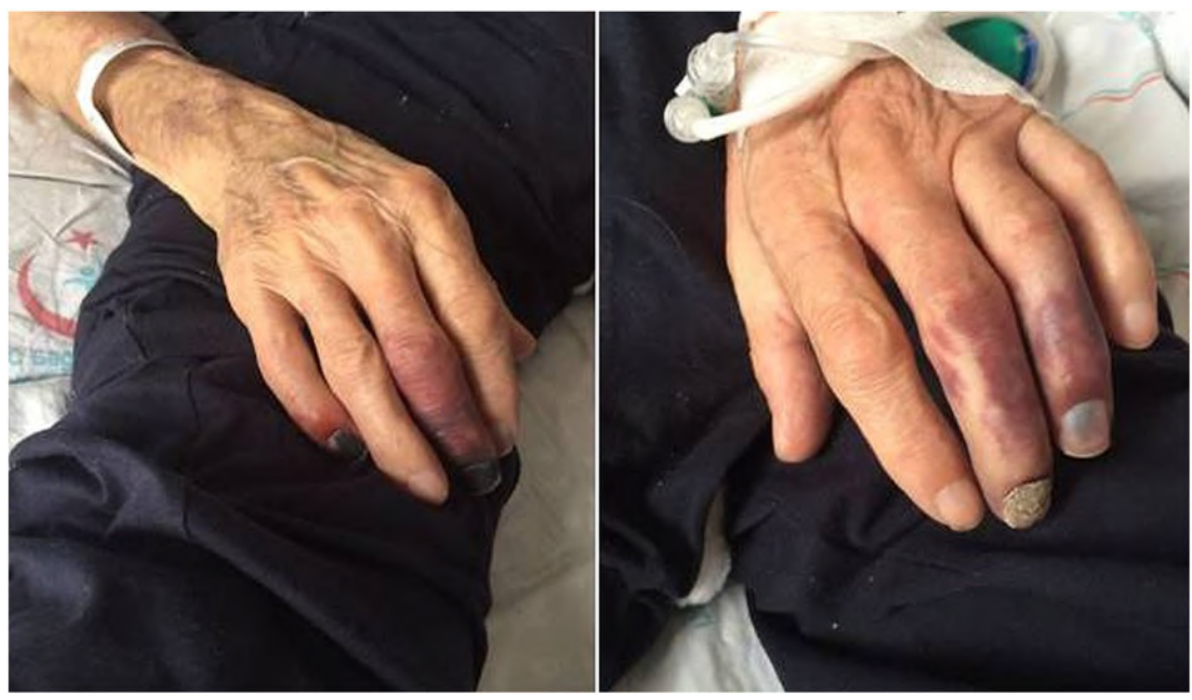

Fig. 1. Clinical image of purple coloured skin manifestation on both of the hands.

outpatient follow-up, skin lesion healed over two months. The serum creatinine level of the patient decreased to $1.9 \mathrm{mg} / \mathrm{dL}$ three weeks after. Six month after the admission, the patient remains asymptomatic with a creatinine level of $1.33 \mathrm{mg} / \mathrm{dL}$ and a serum urea level of $64 \mathrm{mg} / \mathrm{dL}$. To date, there has been no adverse event with the patient.

\section{Discussion}

We hereby described a case of CES with renal and cutaneous involvement affecting extremities after performing coronary intervention in patient with acute myocardial infarction. This case was unique in that cutaneous lesions were observed both on lower extremities and also upper extremities.

CES is a systemic disorder that can spread to multiple organs resulting renal failure, heart failure, gastrointestinal ischemia and skin necrosis. Most affected organs related to CES are the brain, the kidney, the gastrointestinal tract and the skin. Central nervous system involvement may lead to confusion or memory loss. ${ }^{1}$ In our patient, there was no symptom or sign of brain injury. The retinal artery occlusion may present with Hollenhorst plaques on retina examination. Embolization to the kidneys has usually patchy distribution. Elevation of serum

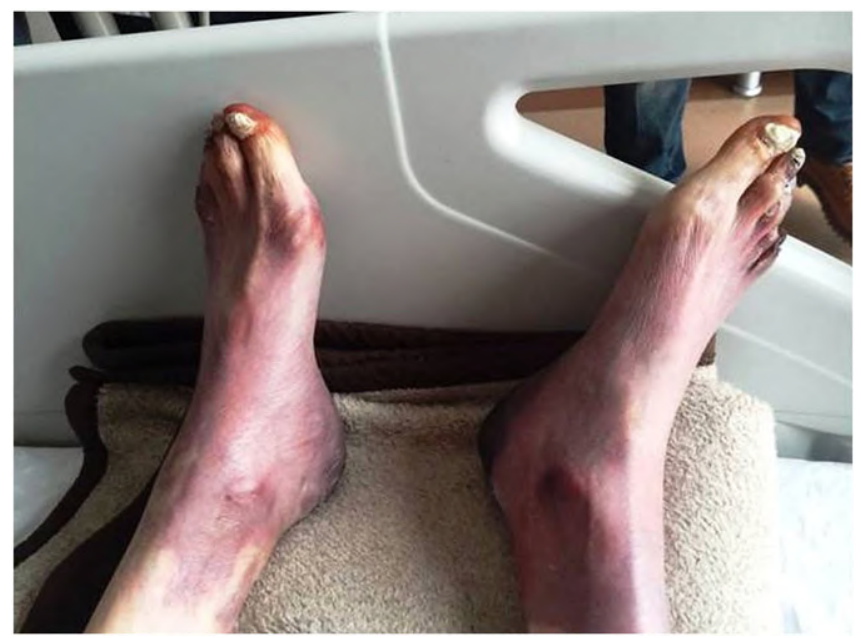

Fig. 2. Clinical image of painful macules on the feet. creatinine and proteinuria are the main laboratory findings. ${ }^{2}$ In the present case, serum creatinine level increased progressively but with supportive care not reached to end-stage renal disease. Cutaneous manifestations of CES include livedo reticularis, gangrene, cyanosis, nodules, macule, ulceration and purpura. Purple or blue discoloration of the foot lead to call ' blue toe syndrome ' synonymous with CES. Cutaneous manifestations almost always located to the lower extremities and rarely extended to upper extremities. ${ }^{3}$ In our case bluish lesions were observed not only on the feet but also on the hands. Risk factors for CES are male gender, smoking, advanced age, hypertension and aortic aneurysm. Development of clinical event is triggered by invasive procedures of aorta, anticoagulation and thrombolysis. ${ }^{4}$ Mainly, a plaque located at proximal, large-calibre artery is ruptured by iatrogenic trauma such as catheter manipulation or spontaneously. Embolization of plaque debris to small or medium sized arteries leads to mechanical occlusion and foreign body inflammatory response which result in end-organ damage. Common source of atheroembolism is the abdominal aorta thus embolism to upper extremities is unusual. CES during coronary angioplasty is uncommon with the incidence of $0.6 \%{ }^{5}$

In the diagnosis of CES, constitutional symptoms and signs are frequent in addition to specific end-organ damage presentation. Leukocytosis with hypereosinophilia, arise in erythrocyte sedimentation rate and C-reactive protein and a decrease in serum complement level may be seen in laboratory tests. Biopsy for any target organ such as kidney or skin could also be obtained for histopathological confirmation. However, none of the test is specific for CES so clinical suspicion is very crucial in the diagnosis of CES. Skin biopsy was not performed in this case. Although pathological findings may be seen in cases, cholesterol cleft identification is limited. Furthermore, in delayed biopsies necrotizing vasculitis may also be seen. ${ }^{6}$ In differential diagnosis, sepsis, disseminated intravascular coagulation (DIC) and heparin-induced thrombocytopenia (HIT) should also keep in mind. While the prolonged activated partial thromboplastin time and prothrombin time and also low levels of protein-C and antithrombin are associated with DIC, coagulation tests is usually normal in HIT with increase in platelet count after cessation of heparin. Positive blood culture and positive sepsis criteria are main determinants in sepsis without DIC. As CES is a clinical entity, in this case diagnosis of CES is confirmed by using symptoms, signs, laboratory findings and close chronological association with coronary intervention.

There is no widely acceptable treatment for CES. Supportive therapy is aimed at end-organ damage. Risk factor modification for 
atherosclerosis should always be considered such as smoking, hypertension, diabetes mellitus and hyperlipidemia. Corticosteroid use may control the inflammation. Statins are considered as key therapy for stabilising atherosclerotic plaque. Antiplatelet agents, reninangiotensin system blockers should be considered for therapy. Surgical repair of the diseased aorta should also be used for therapy. ${ }^{7}$ In our patient, antiplatelet therapy maintained due to acute myocardial infarction and coronary stenting. Oral rosuvastatin was administered at a dose of $40 \mathrm{mg} / \mathrm{day}$. The skin lesions improved gradually in two month follow-up. Serum creatinine level was also decreased to $1.9 \mathrm{mg} / \mathrm{dL}$.

High clinical suspicion is critical for the diagnosis of this clinical entity. CES should be considered when the patient has acute renal failure and specific skin lesions in the extremities after an invasive vascular procedure.

\section{References}

1. Kronzon I, Saric M. Cholesterol embolization syndrome. Circulation 2010;122: 631-641.

2. Scolari F, Tardanico R, Zani R, et al. Cholesterol crystal embolism: a recognizable cause of renal disease. Am J Kidney Dis 2000;36:1089-1109.

3. Falanga V, Fine MJ, Kapoor WN. The cutaneous manifestations of cholesterol crystal embolization. Arch Dermatol 1986;122:1194-1199.

4. Funabiki K, Masuoka H, Shimizu H, et al. Cholesterol crystal embolization (CCE) after cardiac catheterization: a case report and a review of 36 cases in the Japanese literature. Jpn Heart J 2003;44:767-774.

5. Johnson LW, Esente P, Giambartolomei A, et al. Peripheral vascular complications of coronary angioplasty by the femoral and brachial techniques. Catheter Cardiovasc Diagn 1994;31:165-172.

6. Grau R. Pseudovasculitis: mechanisms of vascular injury and clinical spectrum. Curr Rheumatol Rep 2002;4:83-89.

7. Belenfant X, Meyrier A, Jacquot C. Supportive treatment improves survival in multivisceral cholesterol crystal embolism. Am J Kidney Dis 1999;33:840-850. 